\title{
The Symbiosis between Collision and Preimage Resistance*
}

\author{
Elena Andreeva ${ }^{1}$ and Martijn Stam ${ }^{2}$ \\ 1 ESAT/SCD - COSIC, Dept. of Electrical Engineering, \\ Katholieke Universiteit Leuven and IBBT \\ elena.andreeva@esat.kuleuven.be \\ 2 Department of Computer Science, University of Bristol \\ Merchant Venturers Building, Woodland Road, Bristol BS8 1UB, United Kingdom \\ stam@cs.bris.ac.uk
}

\begin{abstract}
We revisit the definitions of preimage resistance, focussing on the question of finding a definition that is simple enough to prove security against, yet flexible enough to be of use for most applications. We give an in-depth analysis of existing preimage resistance notions, introduce several new notions, and establish relations and separations between the known and new preimage notions. This establishes a clear separation between domain-oriented and range-oriented preimage resistance notions. For the former an element is chosen from the domain and hashed to form the target digest; for the latter the target digest is chosen directly from the range.

In particular, we show that Rogaway and Shrimpton's notion of everywhere preimage resistance on its own is less powerful than previously thought. However, we prove that in conjunction with collision resistance, everywhere preimage resistance implies 'ordinary' (domain-based) preimage resistance. We show the implications of our result for iterated hash functions and hash chains, where the latter is related to the Winternitz one-time signature scheme.
\end{abstract}

\section{Introduction}

This paper sheds fresh light on an old topic, namely how to define the basic security properties of cryptographic hash functions. Traditionally, three notions are most often considered: collision resistance, preimage resistance, and second-preimage resistance [14]. A major step towards formal notions was made by Rogaway and Shrimpton [18], who pointed out that the informality usually encountered can be problematic. For instance, it is well-known that collision resistance for unkeyed hash functions is difficult to formalize: an adversary could have a collision for the hash function hard coded in its program. Hence the need for keyed hash functions and a definition of preimage resistance that takes keying into account (where the keys are always publicly known).

\footnotetext{
* This work has been funded in part by the IAP Program P6/26 BCRYPT of the Belgian State (Belgian Science Policy), in part by the European Commission through the ICT program under contract ICT-2007-216676 ECRYPT II, and in part by the Research Council K.U.Leuven: GOA TENSE. The first author is funded by a Ph.D. Fellowship from the Flemish Research Foundation (FWO-Vlaanderen). The work was initiated when the second author was at LACAL, EPFL (Switzerland).
} 
But should preimage resistance hold for all keys, or just for randomly chosen ones? And how is the challenge digest chosen? Rogaway and Shrimpton opted for two different alternatives: the 'strong' notion requires that finding preimages should be hard for all points in the codomain, whereas for the 'weaker' notion a point is chosen uniformly at random from (a subset of) the domain, hashed, and the resulting digest is the challenge to the adversary (who also gets to see the key). With this notion of a random digest, the following three notions are considered:

preimage resistance Both key and challenge digest are random.

everywhere - All challenge digests should be hard to invert under a random key.

always - For all keys, a random challenge digest should be hard to invert.

In total, Rogaway and Shrimpton present 7 security notions for a hash function (1 collision resistance, 3 preimage resistance and 3 second-preimage resistance) and examine the relations between the various notions. The framework thus set up has proved immensely fertile.

For many applications, such as password file hashing, (one-time) digital signatures, key derivation, pseudorandom number generation, etc. an element in the domain is somehow generated (as part of a protocol) and subsequently it needs to be hashed in a preimage-resistant fashion. We will refer to this as domain-oriented preimage resistance. However, different protocols will be based on different distributions. For instance, when passwords are being hashed, it is unrealistic to expect a uniform distribution over the passwords [22] and for Winternitz's one-time signature scheme [13] the use of hash chains means that elements are hashed repeatedly!

Consequently, it is unclear for which distribution or perhaps even distributions (over the domain) the hash function should be proven secure: a notion of preimage resistance is desired that implies all the others. The notion of everywhere preimage resistance was originally believed to serve this purpose. The idea behind it is that the hash function should be preimage resistant for all, possibly induced, distributions on the digest. Indeed, Rogaway and Shrimpton [18, Proposition 6, (6)] claim that everywhere preimage resistance implies (domain-oriented) preimage resistance, but refrain from giving a proof since "the implication is straightforward" and "can easily be verified." The notion has also been used directly, for instance a positive result has been obtained by Neven et al. [15], who relate the security of efficient Schnorr signatures with compact group representations (such as elliptic curves) to the underlying everywhere preimage security of the hash function.

When designing a hash function it is therefore natural to restrict to proving everywhere preimage resistance and conclude this suffices for other notions of preimage resistance as well. Indeed, this approach has been adopted by the MD6 hash function team [17] and the revisiting of the PGV/BRS hash functions by Stam [21] (here Black et al. [4] already showed security with respect to other notions of preimage resistance, cf. [5]). Unfortunately, as we will show, the claim that everywhere preimage resistance implies the practitioner's variety is incorrect. The (contrived) hash function $\mathcal{H}_{K}(X)=K$ is unconditionally everywhere preimage resistant but fails to be meaningfully preimage resistant. It is this simple observation that has prompted this work: a renewed quest for the 'right' notion of preimage resistance. 
Our contribution. As Rogaway himself puts it [19, $\triangleright 15]$, "Definitions emerge, change, and die more than people think." In this paper we revisit the classic definitions of Rogaway and Shrimpton, where our focus will be on determining a notion of preimage security for keyed hash functions that is simple enough to prove security against, yet flexible enough to be of use for most applications.

To reflect the two basic possibilities of selecting target digests-letting it be induced from a distribution over the domain of the hash function, or picking it directly from a distribution over its codomain-we introduce some new terminology in Section 3. In the first scenario, we will speak of a domain-oriented notion; this also includes a generalization of always preimage resistance. In the second scenario, we speak of a range-oriented notion, which includes everywhere preimage resistance.

As already mentioned, we will allow arbitrary distributions (of finite support) to generate the challenge digest, instead of only equidistributed bitstrings of a given length as in the original [18]. Moreover, for all notions we will impose the same interface on the adversary. This minor change in the syntax of the various preimage-resistance definitions compared to Rogaway and Shrimpton allows us, for a single adversary, to discuss the preimage-finding advantage relative to any of the notions. In Section 4 we couple this with a new description of the preimage-finding advantages in terms of matrices in order to prove implications between notions by simple linear algebra. Here we also include several new notions of preimages resistance to make our reexamination as comprehensive as possible.

However, the aforementioned counterexample $\mathcal{H}_{K}(X)=K$ (which is unconditionally everywhere preimage resistant) serves to show that everywhere preimage resistance does not imply any meaningful form of domain-oriented preimage resistance. Section 5 is devoted to this and other separations to explore the relationship between domainoriented preimage resistance and range-oriented preimage resistance.

Our main contribution (Section 6) is a restoration of everywhere preimage resistance as the notion. We prove that if a hash function is both collision resistant and everywhere preimage resistant, then it is also domain-oriented preimage resistant, with the obvious caveat that the distribution over the domain needs to have sufficient entropy (more precisely, we require the Rényi ${ }_{2}$-entropy of the input to be significantly higher than the output bitlength of the hash function).

Of course, it was already known that-for sufficiently compressing functionscollision resistance implies preimage resistance [18], but this is a result of limited use. For instance, if a hash function outputs $n$ bits, this (old) implication cannot guarantee preimage resistance beyond collision resistance. Put concretely, this approach cannot prove preimage resistance beyond $\mathcal{O}\left(2^{n / 2}\right)$ due to the birthday bound. In contrast, our result shows that when you combine collision and everywhere preimage resistance, much stronger claims are possible. Indeed, if both collision and everywhere preimage resistance are optimal, we can claim near optimal domain-oriented preimage resistance, so up to $\mathcal{O}\left(2^{n}\right)$ hash function calls. We stress that our bound does have a loss getting there: the advantage against domain-oriented preimage resistance is roughly upper bounded by the square root of the advantage against everywhere preimage resistance.

In Section 7 we investigate the implications of our results for the security of iterated hash functions. This unearths an hitherto unsuspected symbiosis between collision 
resistance and everywhere preimage resistance. It was already known that the plain Merkle-Damgård iteration (so without length strengthening) preserves collision resistance provided it is hard to find a preimage for the initial vector. But the latter is implied by everywhere preimage resistance, so here everywhere preimage resistance aids collision resistance. On the other hand, for our new result it is just the other way around, with collision resistance lending a helping hand to preimage resistance.

Contrary to domain-oriented notions, everywhere preimage resistance itself is preserved rather easily by an iterative hash function [1]. In fact, we will introduce the concept of dominance-a strong form of property preservation - and show that everywhere preimage resistance is dominant, meaning that everywhere preimage resistance of the full iterated hash function is inherited from only the last call in the iteration. (If that one is everywhere preimage resistant, then so is the entire hash function.) This allows us to conclude that if a compression function is collision resistant and the last (possibly distinct) call is collision resistant and everywhere preimage resistant, the full iterated hash function (strengthened or not), has excellent preimage resistance for all input distributions of sufficient Rényi ${ }_{2}$-entropy.

With only minor modifications, our results apply to hash chains as well, where typically a modified version of preimage resistance is used. Informally, given $K$ and $Y=\mathcal{H}_{K}^{n}(X)$ it should be hard to come up with $X^{\prime}$ such that $\mathcal{H}_{K}^{1}\left(X^{\prime}\right)=Y$ (where the superscripts denote repeated application). Hash chains are used for instance in Winternitz's one-time signature scheme [13]. It turns out that this modified version of preimage resistance is also implied by a combination of collision and everywhere preimage resistance. Previously $[9,12]$ it was only known how to prove the Winternitz scheme secure with the additional property of 'undetectability' (essentially the property a pseudorandom generator has, but without imposing expansion) or using a non-standard property called quasi-inversion [10].

It should be noted that our results (Theorems 14 and 19) are stated in the standard model with a crucial reliance on the hash function being keyed, where the keying is needed to give formal meaning to both notions used (everywhere preimage resistance and collision resistance). This begs the question what can be said about practical, unkeyed hash functions (for instance in the context of the SHA-3 competition). While collision resistance still has informal meaning (cf. Rogaway's human ignorance approach [20]), there seems no natural interpretation of everywhere preimage resistance for fixed-key hash functions. Fortunately, when we consider fixed-key hash functions in the ideal (or unpredictable) cipher model, the random choice of the blockcipher can, to some extent, be regarded as taking on the role of the key. As our results also apply to these oracle-based models, we can still derive meaningful results for many fixed-key hash functions (albeit no longer in the standard model). Indeed, we often use examples based on the ideal cipher model to illustrate our theorems. The main reason is that proofs in the ideal cipher model are the richest source of concrete bounds on collision and everywhere preimage resistance, as needed for the application of our theorems.

Finally, Rogaway and Shrimpton [18] also discuss the relationship between preimage resistance and the other two standard notions of collision resistance and secondpreimage resistance. Since second-preimage definitions are intrinsically domain-oriented, for these everywhere second-preimage resistance does imply the other notions. 


\section{Preliminaries}

Hash functions. We consider keyed hash functions, that is a mapping $\mathcal{H}: \mathcal{K} \times \mathcal{X} \rightarrow \mathcal{Y}$ for finite sets $\mathcal{K}, \mathcal{X}$, and $\mathcal{Y}$. Given the finite set $\mathcal{K}$, we write $K \stackrel{\$}{\leftarrow} \mathcal{K}$ for sampling uniformly at random: for all $k \in \mathcal{K}$ it holds that $\operatorname{Pr}[K=k]=|\mathcal{K}|^{-1}$. For (finite) set $\mathcal{X}$, we will use $X$ to denote a random variable over that set and we use $\mathbf{p}=\left(p_{x}\right)_{x \in \mathcal{X}}$ for its distribution. In particular, if $X \stackrel{\&}{\leftarrow} \mathbf{p}$ then for all $x \in \mathcal{X}$ we have that $\operatorname{Pr}[X=x]=p_{x}$. The set $\mathcal{Y}$, random variable $Y$ and distribution $\mathbf{q}$ have a similar relationship. Let $\mathbf{u}$ denote a uniform distribution (either over $\mathcal{X}$ or $\mathcal{Y}$, depending on the context). In some cases it can be useful to allow an infinite, yet countable $\mathcal{X}$ (or even $\mathcal{Y}$ ), in this case we require the support of $\mathbf{p}$ (resp. $\mathbf{q}$ ), i.e. the set of $x \in \mathcal{X}$ for which $p_{x}>0$, to be finite.

A distinction can be made between standard model hash functions and hash functions based on primitive, such as a blockcipher, that is later modelled as ideal in a proof. If we let $\operatorname{Block}(\kappa, n)$ denote the set of all blockciphers with $\kappa$-bit key and operating on $n$-bit blocks (for positive integers $\kappa$ and $n$ ) then a blockcipher-based hash function is a mapping $\mathcal{H}: \operatorname{Block}(\kappa, n) \times \mathcal{X} \rightarrow \mathcal{Y}$ given by a program that, given $x \in \mathcal{X}$, computes $\mathcal{H}^{E}(x)$ via access to an oracle $E \in \operatorname{Block}(\kappa, n)$ modelling an (ideal) blockcipher (here we use the convention to write oracles that are provided to an algorithm as superscripts). Essentially, $\mathcal{K}=\operatorname{Block}(\kappa, n)$ and the blockcipher takes on the role of key. However, given its long description length, it is never spelled out explicitly and only ever used in a black-box manner.

Attacks and adversaries. An adversary is an algorithm that has to break some welldefined property of the hash function. Typically the adversary consumes resources (time, space, queries). Our convention will be that, for standard model hash functions, the only resource that counts is the runtime of the algorithm, when run on a universal machine. If the hash function is based on an idealized primitive, it is customary to consider information-theoretic adversaries only. Information-theoretic adversaries are computationally unbounded and their complexity is measured only by the number of queries made to their oracles. Hence, in this case we will count the number of queries, denoted $q$, as resource (instead of time).

For hash function $\mathcal{H}$, property $\mathrm{xxx}$ and adversary $\mathcal{A}$ the advantage is denoted $\operatorname{Adv}_{\mathcal{H}}^{\mathrm{xx}}(\mathcal{A})$. The advantage will always be a real number between 0 and 1 (where 0 is ultimate security and 1 signals complete success for the adversary). For standard model hash functions, we will write $\operatorname{Adv}_{\mathcal{H}}^{\times \times x}(t)$ for the maximum advantage that can be attained over all adversaries using at most $t$ resources. When describing attacks on real-world hash functions, it is customary to scale the time relative to the time it takes to evaluate the hash function (or rather the compression function in the typical case of an iterated hash). We will write $t_{q}$ to make this explicit (so $t_{q}=q t_{1}$, where $t_{1}$ corresponds to the time to evaluate the compression/hash function once). We will say that a hash function is $(t, \epsilon)$ $\mathrm{XXX}$-secure if $\operatorname{Adv}_{\mathcal{H}}^{x \times x}(t) \leq \epsilon$. For the ideal cipher model we switch to $\operatorname{Adv}_{\mathcal{H}}^{x \times x}(q)$ for the maximum advantage that can be attained over all adversaries using at most $q$ queries (and talk of $(q, \epsilon) \times x \times$-secure).

For instance, a collision-finding adversary is an algorithm whose goal it is to find collisions in some specified compression or hash function. 
Definition 1. Let $\mathcal{H}: \mathcal{K} \times \mathcal{X} \rightarrow \mathcal{Y}$ be a hash function. The collision-finding advantage of adversary $\mathcal{A}$ is defined to be

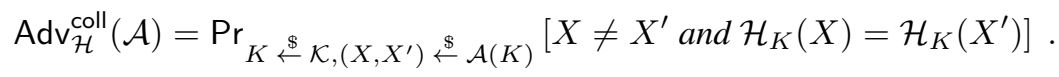

Rényi $_{2}$-entropy, collision probability and balance measure. Let $X$ be a random variable defined over a finite set $\mathcal{X}$ with distribution $\mathbf{p}$. Then the collision probability $P_{2}(X)$, the Rényi $i_{2}$-entropy $H_{2}(X)$, and the balance $\mu(X)$ of $X$ are defined as

$$
P_{2}(X)=\sum_{x \in \mathcal{X}} p_{x}^{2}, \quad H_{2}(X)=-\lg \left(\sum_{x \in \mathcal{X}} p_{x}^{2}\right), \quad \mu(X)=-\log _{|\mathcal{X}|}\left(\sum_{x \in \mathcal{X}} p_{x}^{2}\right) .
$$

From these definitions it is easy to verify that $H_{2}(X)=-\lg P_{2}(X)$ and $\mu(X)=$ $H_{2}(X) / \lg |\mathcal{X}|$. (Bellare and Kohno [3] introduced the balance $\mu$, apparently not aware of the link with collision probability and Rényi ${ }_{2}$-entropy; we thank Krzysztof Pietrzak and Stefano Tessaro for pointing out this link [7].) We will abuse notation and write $P_{2}(\mathbf{p})$ as shorthand for $P_{2}(X)$ where $X$ is a random variable with distribution $\mathbf{p}$.

\section{Defining Preimage Resistance}

A preimage-finding adversary is an algorithm whose goal it is to find preimages for some specified hash or compression function. There exist several definitions depending on the distribution of the element for which a preimage needs to be found. Rogaway and Shrimpton [18] define three basic notions: standard, everywhere, and always preimage resistance.

Existing security notions. Everywhere preimage resistance (epre) formally states that any point in the range is hard to invert: it is meant to capture the idea that preimage resistance holds with respect to any message distribution.

We relabel the standard preimage notion as domain-oriented preimage resistance (dpre). To have a meaningful definition in case of an infinite domain, Rogaway and Shrimpton measure the preimage advantage relative to an additional parameter, denoting the length of the input used to generate the challenge digest. Moreover, all inputs of the same length are equally likely, i.e. the input to be hashed is chosen from a uniform distribution. However, as already noted, the input to the hash function does not always originate from a uniform distribution. For this reason, we extend the notion for any arbitrary finitely-supported distribution $\mathbf{p}$ over the domain. More precisely, our domain-oriented preimage resistance is defined with respect to a distribution over the range induced by the (uniformly random) key selection and a given fixed distribution $\mathbf{p}$ over the domain space.

The third notion, always preimage resistance (apre), requires a hash function to be preimage resistant for any fixed key. Here originally the challenge digest is chosen by hashing a uniformly chosen input of prescribed bitlength. Similarly to the dpre notion, we will allow an arbitrary distribution $\mathbf{p}$ over the domain instead. 
Together with these three notions, we recall another popular notion of preimage resistance [4], namely relative to a target digest chosen uniformly at random. Again, we allow any distribution $\mathbf{q}$ over the range instead, as long as it is independent of the key. We will refer to this notion as range-oriented preimage resistance (rpre).

Apart from the differences in our definitions outlined above, we introduce another change compared to those given by Rogaway and Shrimpton. For all notions, we input both the key and the target digest to the adversary. This change is purely syntactical and, for everywhere preimage resistance feeding the adversary a target digest is strictly superfluous. However, our approach has the important advantage that it unifies the adversarial interfaces for the various preimage notions: for a single adversary, we can consider its advantage with respect to any of the notions mentioned. This simplifies proving relations and separations between notions.

Note that for the apre and dpre notions the purported preimage $X^{\prime}$ returned by the adversary need not be part of p's support. All notions bar apre can also be meaningfully defined in the ideal cipher model (the reason that apre does not make sense is that it would essentially require security for all possible instantiations of the blockcipher, so no security can be derived from the cipher being ideal). For always and everywhere preimage resistance alternative (equivalent) definitions are known based on a two-stage adversary.

Definition 2. Let $\mathcal{H}: \mathcal{K} \times \mathcal{X} \rightarrow \mathcal{Y}$ be a hash function. Let $\mathbf{q}$ be a distribution over $\mathcal{Y}$ and $\mathrm{p}$ a distribution over $\mathcal{X}$. The everywhere, domain-oriented, always and range-oriented preimage finding advantage of adversary $\mathcal{A}$ are defined to be

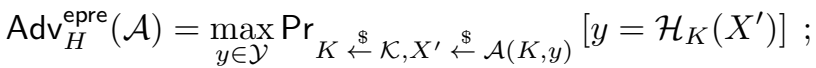

$$
\begin{aligned}
& \operatorname{Adv}_{\mathcal{H}}^{\text {p-dpre }}(\mathcal{A})=\operatorname{Pr}_{K} \stackrel{\&}{\leftarrow} \mathcal{K}, X \stackrel{\&}{\leftarrow} \mathbf{p}, Y \leftarrow \mathcal{H}_{K}(X), X^{\prime} \stackrel{\leftrightarrow}{\leftarrow} \mathcal{A}(K, Y)\left[Y=\mathcal{H}_{K}\left(X^{\prime}\right)\right] ; \\
& \operatorname{Adv}_{\mathcal{H}}^{\text {p-apre }}(\mathcal{A})=\max _{k \in \mathcal{K}} \operatorname{Pr}_{X} \stackrel{\&}{\leftarrow} \mathbf{p}, Y \leftarrow \mathcal{H}_{k}(X), X^{\prime} \stackrel{\$}{\leftarrow} \mathcal{A}(k, Y)\left[Y=\mathcal{H}_{k}\left(X^{\prime}\right)\right] ;
\end{aligned}
$$

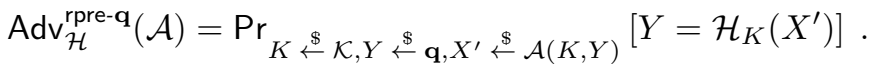

Security notions for non-surjective hashes. For the dpre and apre notions the adversary is guaranteed a challenge for which the digest is in the hash's range for the given key. For the epre and rpre notion this is not necessarily true. The effective range of the hash function can depend strongly on the key. If we define $\mathcal{H}_{k}=\{y \in \mathcal{Y}$ : $\exists x$, s.t. $\left.\mathcal{H}_{k}(x)=y\right\}$, then, given a specific digest $y$ in the codomain, it is possible that after choosing a key $k$ it turns out that $y \notin \mathcal{H}_{k}$ (in other words there exists no input $x$ such that $\mathcal{H}_{k}(x)=y$, or $y$ is an 'invalid' range point). Invalid range points do not have preimages and consequently keep an adversary's advantage unnaturally low. To counter this undesired artefact in the definition, we will credit an adversary for successfully determining $y \notin \mathcal{H}_{k}$. This is what is captured by the epre' and rpre' (plus later spre' ${ }^{\prime}$ ) notions, where $\perp$ is the symbol output by the adversary to signify $y \notin \mathcal{H}_{k}$. We note that this does potentially introduce another artefact, namely an adversary always outputting $\perp$ being deemed successful for a hash function with extremely sparse range. In particular, for expanding functions this definition of epre' and rpre' is not meaning- 
ful. However, for compressing functions the notions are meaningful, and stronger than a possible notion eliminating this second artefact (cf. the upcoming spre' definition).

Definition 3. Let $\mathcal{H}: \mathcal{K} \times \mathcal{X} \rightarrow \mathcal{Y}$ be a hash function. Let $\mathbf{q}$ be a distribution over $\mathcal{Y}$. The boosted range-oriented and boosted everywhere preimage finding advantage of adversary $\mathcal{A}$ are defined to be

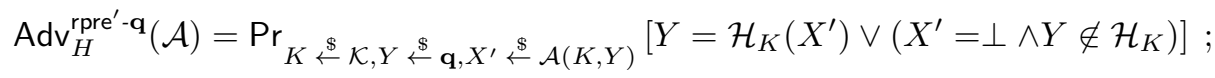

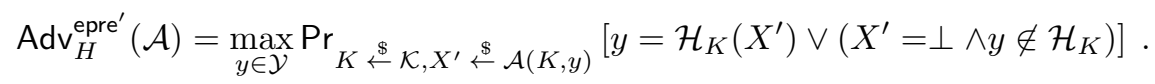

Note that adversaries against the ordinary notions and the boosted notions can still be considered to have identical interfaces; for hash functions that are surjective for all keys the ordinary notion and the corresponding boosted notion collapse.

Somewhere preimage resistance: a notion of insecurity. We also give the natural but novel definition of somewhere preimage security (spre). It is the opposite of the epre notion and it captures the intuition that there is some point in the range that is hard to invert.

This definition is typically too weak to use for any application, but it often best captures the successful adversary's capabilities in the sense that it gives a lower bound on the preimage advantage of an adversary. Its main use is therefore as measure of insecurity. As mentioned above, for sparse hash functions an adversary always outputting $\perp$ will be deemed successful for the spre' notion. Because we want to use this notion for insecurity, we will weaken the notion by only considering eligible adversaries: an adversary is eligible iff it never incorrectly outputs $\perp$. (Of course spre is an even weaker notion, but certain indisputably insecure hash functions manage to be spre-secure making the notion less suitable.)

Definition 4. Let $\mathcal{H}: \mathcal{K} \times \mathcal{X} \rightarrow \mathcal{Y}$ be a hash function. The somewhere and boosted somewhere preimage finding advantage of adversary $\mathcal{A}$ are defined to be

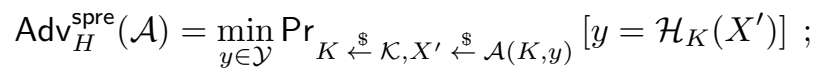

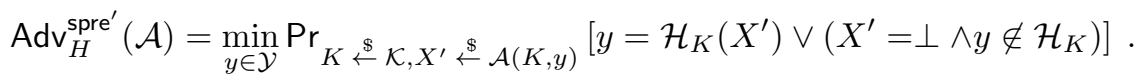

where for spre' only adversaries $\mathcal{A}$ are considered for which

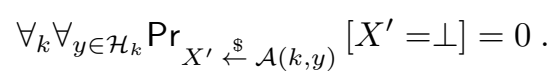

Examples. For integer $n$, let $E \in \operatorname{Block}(n, n)$ and consider the compression function $H^{E}(M, V)=E_{V}(M)$. Its MD-iterate is one of the eight type-II blockcipher-based hash functions as introduced by Black et al. [4] and it is known that the MD-iterated hash function built from it is everywhere preimage resistant up to roughly $2^{n / 2}$ queries. 


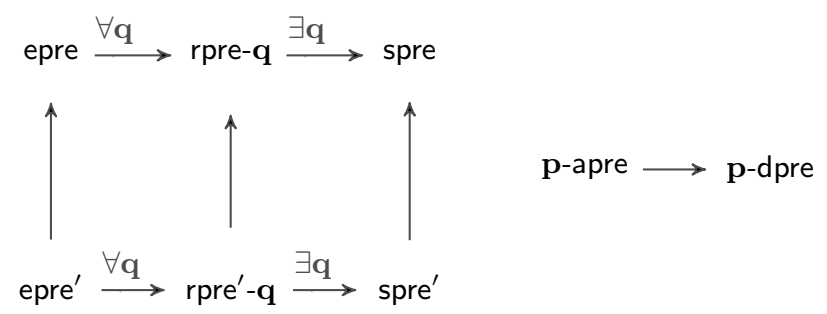

Fig. 1. Overview of implications (Theorem 8)

At the same time, regardless of initial value and target digest, an attacker can find a preimage with roughly $2^{n / 2}$ queries using a meet-in-the-middle attack. Somewhere preimage resistance is the right notion to formally express the success probability of such an adversary. Moreover, since the gap between everywhere and somewhere preimage resistance is small in this case, there is little left to prove: it is fair to say the compression function is preimage resistant up to $2^{n / 2}$ queries.

On the other hand, consider the compression function $H^{F}(M, V)=F(M) \oplus F(V)$, where $F$ is a public random function from $n$-bits to $n$-bits (an adversary only has oracle access to $F$ and not to any inverse). This compression function is not everywhere preimage resistant. Regardless of the choice of $F$, it is easy to find a preimage of the all zero-string $0^{n}$ : any pair $(M, V)$ with $M=V$ will do. However, for all other possible images, finding preimages $i s$ hard (taking roughly $2^{n / 2}$ queries). Consequently, there is a big gap between everywhere and somewhere preimage resistance and for many applications the compression function might still be fine.

\section{Implications between Preimage Notions}

To exhibit the implication and separation results we adhere to the framework set by Rogaway and Shrimpton [18].

Definition 5. Fix $\mathcal{K}, \mathcal{X}$ and $\mathcal{Y}$. A security notion $\mathrm{xxx}$ implies a different security notion yyy (to $\epsilon$ ), or $\mathrm{xxx} \rightarrow$ yyy (to $\epsilon$ ), if for all hash functions $\mathcal{H}: \mathcal{K} \times \mathcal{X} \rightarrow \mathcal{Y}$ the adversarial advantage $\operatorname{Adv}_{\mathcal{H}}^{\mathrm{yyy}}(t) \leq c \operatorname{Adv}_{\mathcal{H}}^{\times \times \times}\left(t^{\prime}\right)(+\epsilon)$ for some absolute constant $c$ and $t^{\prime} \leq t+$ cTime T. $_{\text {. }}$

When $\epsilon=0$, the implication is called conventional, otherwise it is called provisional. The implications we present below are all conventional, in fact, they are even stronger. They are what we call universal, denoted $\mathrm{xxx} \Rightarrow \mathrm{yyy}$ : for all eligible adversaries $\mathcal{A}$ it holds that $\operatorname{Adv}_{\mathcal{H}}^{\mathrm{xx}}(\mathcal{A}) \geq \operatorname{Adv}_{\mathcal{H}}^{\mathrm{yyy}}(\mathcal{A})$. A prerequisite for a universal implication between two notions is that the respective adversaries have identical interfaces.

Some of our security notions are distribution dependent. In this case the implication holds for all distributions (see also Theorem 8). Note that logically $\forall \mathbf{p}$ (p-xxx $\Rightarrow$ yyy) is equivalent to $(\exists \mathbf{p} \mathbf{p}$ - $\mathrm{xxx}) \Rightarrow$ yyy whereas $\forall \mathbf{p}$ ( $\mathrm{xxx} \Rightarrow \mathbf{p}$-yyy) is equivalent to $\mathrm{xxx} \Rightarrow$ $(\forall \mathbf{p}$ p-yyy). Finally, if xxx $\Rightarrow$ yyy and yyy $\Rightarrow$ zzz, then also xxx $\Rightarrow$ zzz. 
Using matrices to express advantages. In Lemma 7 we reframe the various advantages of $\mathcal{A}$ in terms of two matrices (proof in the full version).

Definition 6. Let $\mathcal{A}$ be an adversary against preimage resistance, then the associated success probability matrix $\mathrm{A} \in[0,1]^{|\mathcal{K}| \times|\mathcal{Y}|}$ is given by (defined entry-wise where $k \in$ $\mathcal{K}, y \in \mathcal{Y})$ :

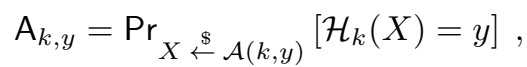

where the probability is taken over the random coins of $\mathcal{A}$. For the boosted notions:

$$
\mathrm{A}_{k, y}^{\prime}=\operatorname{Pr}_{X \stackrel{\&}{\leftarrow}(k, y)}\left[\mathcal{H}_{k}(X)=y \vee\left(X=\perp \wedge y \notin \mathcal{H}_{K}\right)\right] .
$$

For hash function $\mathcal{H}: \mathcal{K} \times \mathcal{X} \rightarrow \mathcal{Y}$ and distribution $\mathbf{p}$ over $\mathcal{X}$ we define the associated challenge probability matrix $\mathrm{B} \in[0,1]^{|\mathcal{Y}| \times|\mathcal{K}|}$ by

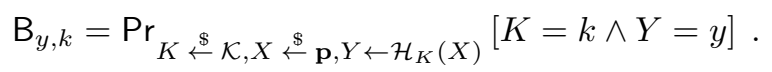

(Since keys are distributed uniformly, $\sum_{y} \mathrm{~B}_{y, k}=1 /|\mathcal{K}|$ for all $k \in \mathcal{K}$.)

Lemma 7. Let $\mathcal{H}$ be a hash function, $\mathbf{q}$ a distribution over $\mathcal{Y}, \mathbf{p}$ a distribution over $\mathcal{X}$ with associated matrix $\mathrm{B}, \operatorname{Tr}(\mathrm{A} \cdot \mathrm{B})=\sum_{k \in \mathcal{K}}(\mathrm{A} \cdot \mathrm{B})_{k, k}$ the trace of the square matrix $(\mathrm{A} \cdot \mathrm{B})_{k, k}$, and $\mathcal{A}$ a preimage-finding adversary with associated matrix $\mathrm{A}$, then

$$
\begin{gathered}
\operatorname{Adv}_{\mathcal{H}}^{\text {p-dpre }}(\mathcal{A})=\operatorname{Tr}(\mathrm{A} \cdot \mathrm{B}), \quad \operatorname{Adv}_{\mathcal{H}}^{\text {p-apre }}(\mathcal{A})=\max _{k \in \mathcal{K}}|\mathcal{K}| \cdot(\mathrm{A} \cdot \mathrm{B})_{k, k} ; \\
\operatorname{Adv}_{\mathcal{H}}^{\text {epre }}(\mathcal{A})=\max _{y \in \mathcal{Y}} \sum_{k \in \mathcal{K}} \mathrm{A}_{k, y} /|\mathcal{K}|, \quad \operatorname{Adv}_{\mathcal{H}}^{\text {spre }}(\mathcal{A})=\min _{y \in \mathcal{Y}} \sum_{k \in \mathcal{K}} \mathrm{A}_{k, y} /|\mathcal{K}| ; \\
\operatorname{Adv}_{\mathcal{H}}^{\text {rpre-q }}(\mathcal{A})=\sum_{y \in \mathcal{Y}} \sum_{k \in \mathcal{K}} q_{y} \mathrm{~A}_{k, y} /|\mathcal{K}| .
\end{gathered}
$$

For epre', rpre', and spre' replace $\mathrm{A}$ with $\mathrm{A}^{\prime}$.

The implications. Below we list all the implications (proof in the full version). Most are straightforward given the matrix representation developed above. The final implication (apre $\Rightarrow$ dpre) was already claimed by Rogaway and Shrimpton (for simplified distributions). We would like to point out a small caveat that seemed to have gone largely unnoticed so far. Against always preimage resistance there is a well-known attack $[2,11]$ running in time $|\mathcal{Y}|^{2 / 3}$, whereas for ordinary preimage resistance the 'desired' level of resistance is roughly $|\mathcal{Y}|$. In other words, optimal always preimage resistance does not imply optimal preimage resistance. (Cf. optimal collision resistance not implying optimal preimage resistance, even for sufficiently compressing hash functions.)

Theorem 8. The following universal implications, as depicted in Figure 1, hold:

1. epre $\Rightarrow$ epre, $\forall \mathbf{q}\left(\right.$ rpre $^{\prime}-\mathbf{q} \Rightarrow$ rpre-q) and spre $^{\prime} \Rightarrow$ spre.

2. epre $\Rightarrow(\forall \mathbf{q}$ rpre-q) and $(\exists \mathbf{q}$ rpre-q) $\Rightarrow$ spre.

3. epre $^{\prime} \Rightarrow\left(\forall \mathbf{q}\right.$ rpre' $\left.^{\prime}-\mathbf{q}\right)$ and $\left(\exists \mathbf{q}\right.$ rpre $\left.^{\prime}-\mathbf{q}\right) \Rightarrow$ spre $^{\prime}$.

4. $\forall \mathbf{p}(\mathbf{p}$-apre $\Rightarrow \mathbf{p}$-dpre)

We note that in fact $(\forall \mathbf{q}$ rpre-q) $\Rightarrow$ epre also holds, making the first parts of 8.2 and 8.3 above equivalences. 


\section{Separations}

As in the work of [18] a separation here denotes a nonimplication. Separations come in two flavours: unconditional and conventional.

For an unconditional separation $x x x \neq y y y$, there exists, without precondition, an $x x x$-secure function that is not yyy-secure. For instance the identity function is collision resistant without the need for further assumptions. Our main, novel observation here is that unconditionally epre-secure hash functions exist, leading to a host of separations (condensed in Theorem 11). In particular, we show that Rogaway and Shrimpton's (unproven) claim that epre $\rightarrow$ dpre is false.

For a conventional separation $x x x \not \rightarrow$ yyy, the starting point is a hash function $\mathcal{H}$ that already is $x x x$-secure. From this one builds a new hash function $\mathcal{H}^{\prime}$ (with the same $\mathcal{K}, \mathcal{X}, \mathcal{Y}$ as $\mathcal{H}$ ) that is still $x \times x$-secure, but demonstrably yyy-insecure. Theorem 12 contains, in addition to separations involving our new notions, also a new (tighter) separation for $\mathbf{u}$-apre $\not \rightarrow$ epre. The counterexample used by Rogaway and Shrimpton [18, Theorem 5 (12)] is in fact erroneous; items (1), (17), (19), (21), (24), (25), and (26) of [18, Theorem 5] can be fixed similarly.

Below we give the formal definitions.

Definition 9. Fix $\mathcal{K}, \mathcal{X}$ and $\mathcal{Y}$ and let security notions $\mathrm{xxx}$ and $\mathrm{yyy}$ be given. Then

- Unconditional separation or $\mathrm{xxx}+$ yyy to $\epsilon$ if there exists a $\mathcal{H}: \mathcal{K} \times \mathcal{X} \rightarrow \mathcal{Y}$ such that $\operatorname{Adv}_{\mathcal{H}}^{\mathrm{xxx}}(t) \leq \epsilon$ for all $t$ and $\operatorname{Adv}_{\mathcal{H}}^{\mathrm{yyy}}\left(t^{\prime}\right)=1$, where $t^{\prime}=c$ Time $_{\mathcal{H}}$ for some absolute constant $c$.

- Conventional separation, or $\mathrm{xxx} \not \rightarrow$ yyy to $\epsilon$ if for any $\mathcal{H}: \mathcal{K} \times \mathcal{X} \rightarrow \mathcal{Y}$ there exists a $\mathcal{H}^{\prime}: \mathcal{K} \times \mathcal{X} \rightarrow \mathcal{Y}$ such that $\operatorname{Adv}_{\mathcal{H}^{\prime}}^{\mathrm{xx}}(t) \leq c \operatorname{Adv}_{\mathcal{H}}^{\times x \times}\left(t^{\prime}\right)+\epsilon$ for all $t$ and yet $\operatorname{Adv}_{\mathcal{H}^{\prime}}$ yyy $\left(t^{\prime}\right)=1$ for some $t^{\prime} \leq t+c$ Time $_{\mathcal{H}}$ for some absolute constant $c$.

Some of the separations follow from known separation and implication results, aided by Lemma 10. We will rely aggressively on this lemma to reduce our workload (interpreting the fourth item as a nonimplication as well). In some cases these 'indirect' separations result in a loss of tightness and a direct, tighter separation might be possible.

Lemma 10. Let $\mathrm{xxx}, \mathrm{yyy}$, and $\mathrm{zzz}$ be security notions, then

$$
\begin{aligned}
& \text { 1. If } \mathrm{xxx}+\text { yyy and } \mathrm{zzz} \rightarrow \text { yyy then } \mathrm{xxx}+\mathrm{zzz} \text {. } \\
& \text { 2. If } \mathrm{xxx} \neq \text { yyy and } \mathrm{xxx} \rightarrow \mathrm{zzz} \text { then } \mathrm{zzz} \neq \mathrm{yyy} \text {. } \\
& \text { 3. If } \mathrm{xxx} \nrightarrow \rightarrow \text { yyy and } \mathrm{zzz} \rightarrow \text { yyy then } \mathrm{xxx} \nrightarrow \rightarrow \mathrm{zzz} \text {. } \\
& \text { 4. If } \mathrm{xxx} \nrightarrow \rightarrow \text { yyy and } \mathrm{xxx} \rightarrow \mathrm{zzz} \text { then } \mathrm{zzz} \rightarrow \text { yyy would imply non-existence of } \mathrm{xxx} \text {. }
\end{aligned}
$$

The separations. To simplify the presentation, we will only consider hash functions that work on bitstrings, so $\mathcal{K}=\{0,1\}^{\kappa}, \mathcal{X}=\{0,1\}^{m}$, and $\mathcal{Y}=\{0,1\}^{n}$. We write 0 for the all-zero bitstring (where the length follows from the context), and $\bar{x}$ can mean either padding with zeroes or chopping bits off, both to the correct, contextual length. (With some extra work, most of our bit-oriented counterexamples can be generalized to arbitrary $\mathcal{K}, \mathcal{X}, \mathcal{Y}$.) 
To warm up, consider the separation dpre $\nrightarrow \rightarrow$ apre to $1 /|\mathcal{K}|$ from Rogaway and Shrimpton [18, Theorem 15 (2)]. We interpret the separation as $\forall \mathbf{p}: \quad$ (p-dpre $\not \rightarrow$ p-apre to $1 /|\mathcal{K}|)$, which can be shown by the original counterexample $\mathcal{H}_{K}^{\prime}(X)=\mathcal{H}_{K}(X)$ unless $K=0$, in which case $\mathcal{H}_{0}^{\prime}(X)=0$.

Theorem 11. Unconditional separations from epre.

1. $\forall \mathbf{p}$ : epre $\not \mathbf{p}$-dpre to $2^{-\min (\kappa, n)}$.

2. epre $\not$ spre $^{\prime}$ to $2^{-\min (\kappa, n)}$.

Proof. Let $\mathcal{H}$ be defined as $\mathcal{H}_{K}(X)=\bar{K}$ for all $K \in \mathcal{K}$ and $X \in \mathcal{X}$.

Consider an epre adversary $\mathcal{A}$. The difficulty in finding preimages for $\mathcal{A}$ is that it can only successfully invert on valid range points $Y$. Here the validity depends strongly on the key $K$ : for any fixed $Y$, the number of keys for which it is in the range is at most $\max \left(1,2^{n-\kappa}\right)$, so the probability (over a uniform choice of the key) that $Y$ is in the range, is at most $2^{-\min (\kappa, n)}$. Thus $\mathcal{A}$ succeeds in inverting a chosen target hash point $Y$ with probability at most $2^{-\min (\kappa, n)}$ and our claim for $\left(\infty, 2^{-\min (\kappa, n)}\right)$ epre-security of $\mathcal{H}$ follows.

1. On the other hand, $\mathcal{H}$ is a $\mathbf{p}$-dpre insecure hash function. A p-dpre adversary $\mathcal{A}^{\prime}$ receives a challenge hash value $Y$ and a key $K \stackrel{\$}{\leftarrow} \mathcal{K}$, where $Y=\mathcal{H}_{K}(X)$ for $X \stackrel{\$}{\leftarrow}$ p. Here $Y=\bar{K}$ as per definition of our counterexample hash function. Thus, $\mathcal{A}^{\prime}$ outputs any arbitrarily chosen message $X^{\prime} \in \mathcal{X}$ which is a valid preimage for $Y=\bar{K}$ under $\mathcal{H}$.

2. We define spre'-adversary $\mathcal{A}^{\prime}$ that, upon receiving challenge digest $Y$ and key $K$ outputs any arbitrarily chosen message $X^{\prime} \in \mathcal{X}$ iff $Y=\bar{K}$ and $\perp$ otherwise. This adversary never incorrectly calls $\perp$, it has spre' -advantage 1 .

Theorem 12. Conventional separations involving epre and epre'.

1. $\forall \mathbf{p}:(\mathbf{p}$-apre $\not \rightarrow$ epre) .

2. $\forall \mathbf{q}:\left(\right.$ rpre' $^{\prime}-\mathbf{q} \not \rightarrow$ epre to $\left.2^{-n}\right)$.

3. $\forall \mathbf{p}:\left(\right.$ epre' $\nrightarrow$ $\rightarrow \mathbf{p}$-apre to $\left.2^{-\kappa}\right)$.

The proofs of Theorems 12-1 and 12-2 can be found in the full version. Theorem 12-3 follows from [18, Theorem 5 (7)]'s counterexample (for epre $\not \rightarrow \mathbf{u}$-apre). Here, we build an epre' secure (instead of an epre secure as in the original work) yet p-apre insecure hash function out of an epre' secure function and derive the same bounds.

A relative separation related to epre' $\not \rightarrow \mathbf{u}$-dpre. Ideally, we would like to show that our new notion of boosted everywhere preimage resistance is strong enough to imply domain-oriented preimage resistance. We show that this is unlikely by giving a conventional separation that does not exactly fit Definition 9. That is, in the full version we show that if an epre' secure hash function exists then any (universal) implication between epre' and u-dpre for hash functions with the same key space and range, but increasingly large domain will get increasingly worse (to the point of quickly becoming meaningless). 


\section{Salvaging Everywhere Preimage Resistance for Practical Applications}

In the previous sections we have seen that everywhere preimage resistance does not imply domain-oriented preimage resistance of any sort. In this section we will salvage everywhere preimage resistance, by showing that under certain natural conditions, it does imply domain-oriented preimage resistance. This has the important consequence that it (again) suffices to show that the hash function is everywhere preimage resistant (plus of course the conditions need to be satisfied).

Consider a hash function and a distribution over its domain, such that for all keys the same distribution over the range is induced. In other words, there is a unique distribution over the range that we can consider, and consequently our previous implication (from everywhere preimage resistance to range-oriented preimage resistance) applies. The problem is how to show that for all distributions over the domain the induced distribution over the range is (approximately) key-independent.

Our approach is simple: we argue that for a collision resistant hash function and a distribution over the domain of sufficient Rényi ${ }_{2}$-entropy, the induced distribution is roughly uniform. It is well-known that, for any hash function, collisions can be found using a generic birthday attack. When the hash function is not regular the generic attack is potentially faster than the birthday bound might indicate. This has been studied in detail by Bellare and Kohno [3]; the theorem below is a rephrasing of [3, Theorem 9.2] with the added twist that the adversary need not sample from the domain uniformly.

Theorem 13. Let $\mathcal{H}: \mathcal{K} \times \mathcal{X} \rightarrow \mathcal{Y}$ be a hash function, let $\mathbf{p}$ be a distribution over $\mathcal{X}$ and let $\mathrm{B}$ be the corresponding challenge probability matrix. Consider the (generic black-box) adversary $\mathcal{A}_{q}[\mathbf{p}]$ that independently samples $q$ domain points according to distribution $\mathbf{p}$, evaluates the hash function (on those $q$ sampled domain points), and outputs a collision if one is found. Then, for all real $0 \leq \alpha \leq 2 \sqrt{2}-2$

$$
\left(\begin{array}{l}
q \\
2
\end{array}\right)\left(\left(1-\alpha^{2} / 4-\alpha\right) \tilde{B}-P_{2}(\mathbf{p})\right) \leq \operatorname{Adv}_{\mathcal{H}}^{\text {coll }}\left(\mathcal{A}_{q}[\mathbf{p}]\right) \leq\left(\begin{array}{l}
q \\
2
\end{array}\right) \tilde{B},
$$

where the lower bounds hold for all

$$
q \leq \alpha \tilde{B}^{-1 / 2}
$$

and

$$
\tilde{B}=|\mathcal{K}| \sum_{k \in \mathcal{K}} \sum_{y \in \mathcal{Y}} \mathrm{B}_{y, k}^{2} .
$$

Theorem 13 gives a lower bound on the success probability of a specific generic adversary against collision resistance in terms of the bias $\tilde{B}$ (related to the regularity of the hash function, defined below) and the number of queries asked by the adversary. As we show below (Lemma 17), with some effort we can also turn this around: given a general upper bound on the collision resistance advantage we can upper bound the bias. We need some further technical Lemmas (15 and 16) to take care of small deviations from the uniform distribution and to formally relate the dpre-advantage to the epre one. Combination of the various lemmas leads to Theorem 14, the main result of our paper. 
Interpretation. Given the bound from Theorem 14 (below), it is perhaps not immediately clear what the consequences are, so we will illustrate with a concrete example. Further examples (related to hash function constructions) can be found in the next section.

Consider the blockcipher-based Davies-Meyer construction $\mathcal{H}^{E}(M, V)=E_{M}(V) \oplus$ $V$, mapping $n+m$ bits to $n$ bits when $E \in \operatorname{Block}(m, n)$. Black et al. [4] already showed that (in the ideal cipher model) this construction is $\mathbf{u}$-dpre and rpre- $\mathbf{u}$ secure, but here we are interested in p-dpre security for arbitrary $\mathbf{p}$. From the results by Black et al. it follows that

$$
\operatorname{Adv}_{\mathcal{H}}^{\text {coll }}(q) \leq \frac{1}{2} q(q-1) /\left(2^{n}-q\right) \quad \text { and } \quad \operatorname{Adv}_{\mathcal{H}}^{\text {epre }}(q) \leq q /\left(2^{n}-q\right)
$$

The minimization of $\operatorname{Adv}_{\mathcal{H}}^{\text {coll }}(q) /\left(\begin{array}{c}q \\ 2\end{array}\right)$ boils down to minimizing $\left(2^{n}-q\right)^{-1}$, so $q=2$ is optimal and allowed as long as $P_{2}(\mathbf{p}) \leq 0.002835-\left(2^{n}-2\right)^{-1}$. Suppose we are given a uniform distribution $\mathbf{p}$ over $\mathcal{X}=\{0,1\}^{m}$, so $P_{2}(\mathbf{p})=2^{-m}$. Then the theorem tells us that

$$
\operatorname{Adv}_{\mathcal{H}}^{\text {p-dpre }}(\mathcal{A}) \leq \sqrt{3\left(1+2^{n-m}\right) q /\left(2^{n}-q\right)}
$$

where we have ignored some small terms. Thus, if $m \geq n$ it takes roughly $2^{n-2}$ queries to obtain a reasonable advantage, which is close to optimal. The same result holds even if $\mathbf{p}$ is not uniform, as long as its Rényi $i_{2}$-entropy is (at least) $m$.

Theorem 14. Let $\mathcal{H}: \mathcal{K} \times \mathcal{X} \rightarrow \mathcal{Y}$ be a hash function and let $\mathbf{p}$ be a distribution over the domain $\mathcal{X}$. Let $\operatorname{Adv}_{\mathcal{H}}^{\text {coll }}\left(t_{q}\right)$ be an upper bound on the collision resistance of $\mathcal{H}$ over all adversaries spending time $t_{q}$ (equivalent to evaluating the hash function $q$ times). Then for all preimage finding adversaries $\mathcal{A}$ it holds that

$$
\operatorname{Adv}_{\mathcal{H}}^{\text {p-dpre }}(\mathcal{A}) \leq \sqrt{3|\mathcal{Y}| \cdot \min \left(\operatorname{Adv}_{\mathcal{H}}^{\text {coll }}\left(t_{q}\right) /\left(\begin{array}{l}
q \\
2
\end{array}\right)+P_{2}(\mathbf{p})\right)} \cdot \sqrt{\operatorname{Adv}_{\mathcal{H}}^{\text {epre }}(\mathcal{A})+\frac{1}{|\mathcal{K}|}}
$$

where the minimum is taken over all $q>1$ for which

$$
q^{2}\left(\operatorname{Adv}_{\mathcal{H}}^{\text {coll }}\left(t_{q}\right) /\left(\begin{array}{l}
q \\
2
\end{array}\right)+P_{2}(\mathbf{p})\right) \leq 0.1134
$$

Proof. Recall the definitions of matrices A and B from Definition 6 (the success probability matrix associated to some adversary $\mathcal{A}$, respectively the challenge probability matrix associated with $\mathbf{p}$ and $\mathcal{H}$ ). From Lemma 7 we have that

$$
\operatorname{Adv}_{\mathcal{H}}^{\text {p-dpre }}(\mathcal{A})=\operatorname{Tr}(\mathrm{A} \cdot \mathrm{B}),
$$

which is what we would like to bound. Lemma 7 also provides a bound on the entries of $\mathrm{A}$ given $\operatorname{Adv}_{\mathcal{H}}^{\text {epre }}(\mathcal{A})$. Lemma15 turns this bound into one on $\operatorname{Adv}_{\mathcal{H}}^{\mathrm{p} \text {-dpre }}(\mathcal{A})$ given epreadvantage and matrix $\mathrm{B}$. This new bound takes the form $\max _{S \in \mathcal{K} \times \mathcal{Y},|S|=w} \sum_{(k, y) \in S} \mathrm{~B}_{y, k}$, that is the maximum sum that can be obtained by summing (at most) $w$ distinct entries of $\mathrm{B}$ (where $w$ depends on the epre-advantage).

In Lemma16 we show how we can bound this maximized sum if we know the bias $\tilde{B}$ of the matrix B. This bias is a combinatorial property (normalized sum of squares) that 
follows from the hash function $\mathcal{H}$ and the input distribution dpre. In general it might be hard to pin down, but in Lemma17 we show how to bound the bias based on the collision resistance of the hash function (this step also introduces the Rényi $i_{2}$-entropy or collision probability $P_{2}(\mathbf{p})$ into the bound).

Combining the three lemmas is straightforward and gives the theorem statement.

Lemma 15. Let hash function $\mathcal{H}: \mathcal{K} \times \mathcal{X} \rightarrow \mathcal{Y}$ be given. Let $\mathcal{A}$ be a preimage-finding adversary, let $\mathbf{p}$ be a distribution over $\mathcal{X}$ and let $\mathrm{B} \in[0,1]^{|\mathcal{Y}| \times|\mathcal{K}|}$ be the induced challenge probability matrix. Then

$$
\operatorname{Adv}_{\mathcal{H}}^{\text {p-dpre }}(\mathcal{A}) \leq \max _{S \in \mathcal{K} \times \mathcal{Y},|S|=w} \sum_{(k, y) \in S} \mathrm{~B}_{y, k},
$$

where $w=|\mathcal{Y}| \cdot\left\lceil|\mathcal{K}| \cdot \operatorname{Adv}_{\mathcal{H}}^{\text {epre }}(\mathcal{A})\right\rceil$.

Proof. Let $\mathrm{A} \in[0,1]^{|\mathcal{K}| \times|\mathcal{Y}|}$ be the success probability matrix associated with $\mathcal{A}$, then,

$$
\operatorname{Adv}_{\mathcal{H}}^{\text {p-dpre }}(\mathcal{A})=\operatorname{Tr}(\mathrm{A} \cdot \mathrm{B})
$$

Therefore it suffices to show that $\operatorname{Tr}(\mathrm{A} \cdot \mathrm{B}) \leq \max _{S \in \mathcal{K} \times \mathcal{Y},|S|=w} \sum_{(k, y) \in S} \mathrm{~B}_{y, k}$. Since the trace is symmetric, we have that $\operatorname{Tr}(A \cdot B)=\operatorname{Tr}(B \cdot A)$, or

$$
\operatorname{Tr}(\mathrm{A} \cdot \mathrm{B})=\sum_{y \in \mathcal{Y}}\left(\sum_{k \in \mathcal{K}} \mathrm{A}_{k, y} \cdot \mathrm{B}_{y, k}\right) .
$$

Let us fix a particular $y$, which fixes a row $\mathrm{B}_{y, k}$ and a column $\mathrm{A}_{k, y}$. Lemma 7 implies

$$
\forall y \in \mathcal{Y} \sum_{k \in \mathcal{K}} \mathrm{A}_{k, y} \leq|\mathcal{K}| \cdot \operatorname{Adv}_{\mathcal{H}}^{\text {epre }}(\mathcal{A})
$$

Given a row $\mathrm{B}_{y,} \in\left(\mathbb{R}_{\geq 0}\right)^{|\mathcal{K}|}$ and integer value $1 \leq B \leq|\mathcal{K}|$, we ask ourselves the question how to maximize

$$
\sum_{k \in \mathcal{K}} \mathrm{A}_{k, y} \cdot \mathrm{B}_{y, k}
$$

subject to the constraints that $\mathrm{A}_{k, y} \in[0,1]$ and $\sum_{k \in \mathcal{K}} \mathrm{A}_{k, y} \leq B$.

This is done by using maximum $(=1)$ weights to the maximal elements of $B$, in other words $A_{k, y}=1$ for $B$ values (and zero elsewhere for that column). Moreover, it is then also clear that for this particular $y$, the contribution to the trace will be the sum of the $B$ largest values in the row $\mathrm{B}_{y, \cdot}$.

Equation 1 allows us to bound $B$ by $\left\lceil|\mathcal{K}| \cdot \operatorname{Adv}_{\mathcal{H}}^{\text {epre }}(\mathcal{A})\right\rceil$. If we sum over all $|\mathcal{Y}|$ possible digests, we get the sum of at most $\left.|\mathcal{Y}| \cdot|| \mathcal{K} \mid \cdot \operatorname{Adv}_{\mathcal{H}}^{\text {epre }}(\mathcal{A})\right\rceil$ entries of $\mathrm{B}$. (To get the lemma statement we drop the requirement on these values needed to be picked equally among the rows.) 
Lemma 16. Let $\mathrm{B} \in[0,1]^{|\mathcal{Y}| \times|\mathcal{K}|}$ be a matrix satisfying $\forall_{k \in \mathcal{K}} \sum_{y \in \mathcal{Y}} \mathrm{B}_{y, k}=1 /|\mathcal{K}|$ and $\tilde{B}=|\mathcal{K}| \cdot \sum_{k \in \mathcal{K}} \sum_{y \in \mathcal{Y}} \mathrm{B}_{y, k}^{2}$, then for all positive integer $w$ :

$$
\max _{S \in \mathcal{K} \times \mathcal{Y},|S|=w} \sum_{(k, y) \in S} \mathrm{~B}_{y, k} \leq \sqrt{\frac{\tilde{B} w}{|\mathcal{K}|}} .
$$

Proof. For this proof, we simply ignore the premise that $\forall_{k \in \mathcal{K}} \sum_{y \in \mathcal{Y}} \mathrm{B}_{y, k}=1 /|K|$. Then we achieve a maximum for $\max _{S \in \mathcal{K} \times \mathcal{Y},|S|=w} \sum_{(k, y) \in S} \mathrm{~B}_{y, k}$ if $\mathrm{B}_{y, k}=0$ for all but $w$ entries. For these $w$ nonzero entries their squares sum up to $\tilde{B} /|\mathcal{K}|$. A maximum sum (over the entries themselves) is obtained by a uniform distribution, ${ }^{3}$ that is $\mathrm{B}_{y, k}=$ $\sqrt{\frac{\tilde{B}}{|\mathcal{K}| w}}$ for these $w$ nonzero values, leading to $\max _{S \in \mathcal{K} \times \mathcal{Y},|S|=w} \sum_{(k, y) \in S} \mathrm{~B}_{y, k} \leq$ $\sqrt{\frac{\tilde{B} w}{|\mathcal{K}|}}$. (Taking the ignored premise into account is likely to provide a tighter bound.)

Lemma 17. Let hash function $\mathcal{H}: \mathcal{K} \times \mathcal{X} \rightarrow \mathcal{Y}$ and distribution $\mathbf{p}$ over $\mathcal{X}$ with corresponding challenge probability matrix $\mathrm{B}$ be given. Define

$$
\tilde{B}=|\mathcal{K}| \sum_{k \in \mathcal{K}} \sum_{y \in \mathcal{Y}} \mathrm{B}_{y, k}^{2} .
$$

Then

$$
\tilde{B} \leq 3 \min \operatorname{Adv}_{\mathcal{H}}^{\text {coll }}\left(t_{q}\right) /\left(\begin{array}{l}
q \\
2
\end{array}\right)+P_{2}(\mathbf{p})
$$

where the minimum is taken over all $q>1$ subject to

$$
q^{2}\left(\operatorname{Adv}_{\mathcal{H}}^{\text {coll }}\left(t_{q}\right) /\left(\begin{array}{l}
q \\
2
\end{array}\right)+P_{2}(\mathbf{p})\right) \leq 0.1134
$$

Proof. Let us first recall the relevant parts of Theorem 13, but rewritten to isolate $\tilde{B}$ (this rewrite assumes $q>1$ ). For adversary $\mathcal{A}_{q}[\mathbf{p}]$ (that independently samples $q$ domain points according to distribution $\mathbf{p}$ ) with advantage $\operatorname{Adv}_{\mathcal{H}}^{\text {coll }}\left(\mathcal{A}_{q}[\mathbf{p}]\right)$, it holds, for all real $0 \leq \alpha \leq 2 \sqrt{2}-2$ and all $q>1$, that

$$
\tilde{B} \leq \frac{\operatorname{Adv}_{\mathcal{H}}^{\text {coll }}\left(\mathcal{A}_{q}[\mathbf{p}]\right) /\left(\begin{array}{l}
q \\
2
\end{array}\right)+P_{2}(\mathbf{p})}{1-\alpha^{2} / 4-\alpha}
$$

provided that

$$
\tilde{B} \leq\left(\frac{\alpha}{q}\right)^{2}
$$

In the ideal cipher model, we know that $\mathcal{A}_{q}[\mathbf{p}]$ makes at most $q$ queries and hence $\operatorname{Adv}_{\mathcal{H}}^{\text {coll }}\left(\mathcal{A}_{q}[\mathbf{p}]\right) \leq \operatorname{Adv}_{\mathcal{H}}^{\text {coll }}(q)$. In the standard model we need to upper bound the time

\footnotetext{
${ }^{3}$ Compare the problem of minimizing the sum of the squares given a constraint on the sum of the elements.
} 
$\mathcal{A}_{q}[\mathbf{p}]$ takes: for simplicity we ignore any overhead to actually find the collision and upper bound the time by $t_{q}$, leading to $\operatorname{Adv}_{\mathcal{H}}^{\text {coll }}\left(\mathcal{A}_{q}[\mathbf{p}]\right) \leq \operatorname{Adv}_{\mathcal{H}}^{\text {coll }}\left(t_{q}\right)$. We can then ensure that the provision 2 is satisfied by requiring that $\alpha$ and $q$ themselves are such that

$$
\frac{\operatorname{Adv}_{\mathcal{H}}^{\text {coll }}(q) /\left(\begin{array}{l}
q \\
2
\end{array}\right)+P_{2}(\mathbf{p})}{1-\alpha^{2} / 4-\alpha} \leq\left(\frac{\alpha}{q}\right)^{2}
$$

or, equivalently,

$$
q^{2}\left(\operatorname{Adv}_{\mathcal{H}}^{\text {coll }}(q) /\left(\begin{array}{l}
q \\
2
\end{array}\right)+P_{2}(\mathbf{p})\right) \leq \alpha^{2}\left(1-\alpha^{2} / 4-\alpha\right) .
$$

Finally $\alpha^{2}\left(1-\alpha^{2} / 4-\alpha\right)$ is maximized for $\alpha=(-3+\sqrt{17}) / 2 \approx 0.561553$ giving value $(71-17 \sqrt{17}) / 8 \approx 0.113401$ and the denominator in $\tilde{B}$ upper bound $(7-\sqrt{17}) / 8 \approx$ 0.359612 .

\section{Preimage Resistance of Hash Function Constructions}

In this section we investigate the preimage resistance of iterated hash functions and hash chains and see how our results fit in. For this we consider a general iteration principle and a version of preimage resistance where an adversary is already deemed successful if it can output a correct intermediate chaining variable (e.g. hash chains). Our restriction to a split in two only is just an abstraction: it does not limit generality and the statement encompasses many existing hash function modes of operation (as explained later).

Definition 18. Let $\mathcal{G}^{H_{1}, H_{2}}: \mathcal{K} \times \mathcal{X} \rightarrow \mathcal{Y}$ be an iterated hash function with underlying compression functions $H_{1}: \mathcal{K} \times \mathcal{X}_{1} \rightarrow \mathcal{Y}_{1}$ and $H_{2}: \mathcal{K} \times \mathcal{Y}_{1} \times \mathcal{X}_{2} \rightarrow \mathcal{Y}$ where $\mathcal{X}=\mathcal{X}_{1} \times \mathcal{X}_{2}$ defined by $\mathcal{G}^{H_{1}, H_{2}}\left(K,\left(X_{1}, X_{2}\right)\right)=H_{2}\left(K, H_{1}\left(K, X_{1}\right), X_{2}\right)$. Let $\mathbf{p}$ be a distribution over $\mathcal{X}$. The intermediate preimage finding advantage of adversary $\mathcal{A}$ is defined to be

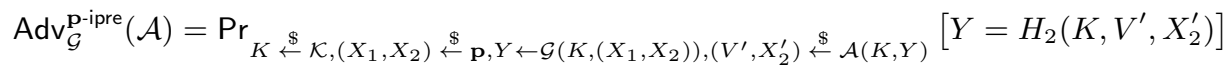

Note that our definition also allows to consider independently keyed hash functions $H_{1}: \mathcal{K}_{1} \times \mathcal{X}_{1} \rightarrow \mathcal{Y}_{1}$ and $H_{2}: \mathcal{K}_{2} \times \mathcal{Y}_{1} \times \mathcal{X}_{2} \rightarrow \mathcal{Y}$ by setting $\mathcal{K}=\mathcal{K}_{1} \times \mathcal{K}_{2}$ and defining $H_{1}^{\prime}$ and $H_{2}^{\prime}$ with keyspace $\mathcal{K}$ (where $H_{1}^{\prime}$ implements $H_{1}$ by ignoring its $\mathcal{K}_{2}$ part of the key; similar for $H_{2}^{\prime}$ ). A similar trick does not work for the domain for several reasons: firstly, we want to consider arbitrary distributions over $\mathcal{X}$ (and these might not all be induced by a 'product' of two distributions); and secondly, ignoring part of the input clearly affects collision resistance (adding and ignoring bits to the key preserves collision resistance, whereas adding and ignoring input bits trivially creates collisions).

In the theorem below we will relate the security of the overall construction $\mathcal{G}$ to that of the underlying compression functions $H_{1}$ and $H_{2}$. Some of it is basic fare, for instance the preservation of collision resistance. Similarly, everywhere preimage security is cleanly preserved for a number of Merkle-Damgård based iterations [1]. However, here we show something stronger, namely that it is in fact dominant. In the context of 
iterated hash functions, we define dominance for a security property, whenever possession of that property by the (very) last compression function $\mathrm{H}_{2}$ implies inheritance by the full, iterated hash function. That is to say, the preservation is valid irrespectively of the cryptographic strength of the compression function $H_{1}$ (or any of the components that make up $H_{1}$ when $H_{1}$ itself for instance has an iterated structure). It turns out that all the unboosted range-oriented preimage resistance notions are dominant. (For the boosted notions, dominance is undercut by the possibility that the effective range for the iterated hash function is much sparser than that of the final compression function.)

Theorem 19. Let $\mathcal{G}$ and $\mathbf{p}$ be given as in Definition 18. Then for any $\mathcal{A}$ there exist equally efficient adversaries $\mathcal{A}^{\prime}$ such that

1. coll is preserved

$$
\operatorname{Adv}_{\mathcal{G}}^{\text {coll }}(\mathcal{A}) \leq \max \left(\operatorname{Adv}_{H_{1}}^{\text {coll }}\left(\mathcal{A}^{\prime}\right), \operatorname{Adv}_{H_{2}}^{\text {coll }}\left(\mathcal{A}^{\prime}\right)\right)
$$

2. ipre implies dpre, or

$$
\operatorname{Adv}_{\mathcal{G}}^{\text {p-dpre }}(\mathcal{A}) \leq \operatorname{Adv}_{\mathcal{G}}^{\text {p-ipre }}\left(\mathcal{A}^{\prime}\right)
$$

3. range-oriented notions of $\mathrm{H}_{2}$ are dominant, i.e. for atk $\in\{$ epre, rpre-q, spre $\}$

$$
\operatorname{Adv}_{\mathcal{G}}^{\text {atk }}(\mathcal{A}) \leq \operatorname{Adv}_{H_{2}}^{\text {atk }}\left(\mathcal{A}^{\prime}\right)
$$

(the statement for rpre-q holds for all distributions $\mathbf{q}$ );

4. together, coll and epre imply ipre. In particular, if $H_{1}$ is collision resistant and $H_{2}$ is both collision resistant and everywhere preimage resistant, then the combined $\mathcal{G}$ is ipre secure. (A concrete statement is provided in the full version.)

Proof (sketch). Items 1 and 2 are standard; for 4, we refer to the full version for details. For 3, given a preimage-finding adversary $\mathcal{A}$ against $\mathcal{G}^{H_{1}, H_{2}}$, we build a preimagefinding adversary $\mathcal{A}^{\prime}$ against $H_{2}$. Let $(K, Y)$ be the challenge of $\mathcal{A}^{\prime}$. It then runs $\mathcal{A}$ on input $(K, Y)$. Suppose $\mathcal{A}$ returns a preimage message $\left(X_{1}^{\prime}, X_{2}^{\prime}\right)$, then $\mathcal{A}^{\prime}$ returns as preimage $\left(H_{1}\left(K, X_{1}^{\prime}\right), X_{2}^{\prime}\right)$. If $\left(X_{1}^{\prime}, X_{2}^{\prime}\right)$ was a valid preimage of $Y$ under $\mathcal{G}$ (so $\left.\mathcal{G}^{H_{1}, H_{2}}\left(K,\left(X_{1}^{\prime}, X_{2}^{\prime}\right)\right)=Y\right)$, then it follows that $\left(H_{1}\left(K, X_{1}^{\prime}\right), X_{2}^{\prime}\right)$ is a valid preimage of $Y$ under $H_{2}$, since $H_{2}\left(K, H_{1}\left(K, X_{1}^{\prime}\right), X_{2}^{\prime}\right)=\mathcal{G}^{H_{1}, H_{2}}\left(K,\left(X_{1}^{\prime}, X_{2}^{\prime}\right)\right)=Y$.

Application to iterated hashes. The generalized Merkle-Damgård transform $[8,14$, 16] is a special case of the split in two. The main design rationale behind the MerkleDamgård construction is the preservation of collision security (coll). More concretely, given a coll-secure compression function, a suffix-free padding function pad and an injective output transformation $g$ (the identity function in the original work of $[8,14]$ ), it is proven that the iterated Merkle-Damgarrd hash function is also coll secure. Alternatively, if pad is just injective and the compression function is both coll and epre, the full hash is also known to be collision resistant. Below the description.

Definition 20. Let $\mathcal{G}^{H}: \mathcal{K} \times \mathcal{C} \times \mathcal{X} \rightarrow \mathcal{Y}$ be a generalized iterative hash function with underlying compression functions $H: \mathcal{K} \times \mathcal{C} \times \mathcal{M} \rightarrow \mathcal{C}$ Let pad : $\mathcal{X} \rightarrow \mathcal{M}^{+}$be a fixed (injective) padding function and $g: \mathcal{C} \rightarrow \mathcal{Y}$ be a fixed output transformation. Then for a key $K \in \mathcal{K}$, initial vector $I V \in \mathcal{C}$ and input $X \in \mathcal{X}$, the digest $Y=\mathcal{G}^{H}(K, I V, X)$ is computed as follows 
1. $V_{0} \leftarrow I V, \mathbf{M} \leftarrow \operatorname{pad}(X) \in \mathcal{M}^{+}$

2. Parse $\mathbf{M}$ as $\left(M_{1}, \ldots, M_{\ell}\right)$

3. For $i=1, \ldots, \ell$ compute $V_{i}=H\left(K, V_{i-1}, M_{i}\right)$

4. Output $Y \leftarrow g\left(V_{\ell}\right)$.

Andreeva et al. [1] suggest that preimage resistance for some Merkle-Damgård based iterations with respect to induced (key-dependent) distributions is not preserved: the induced distribution on the images for the hash function (for long messages) might not actually be induced by any distribution on the domain of the compression function (due to the iteration potentially emphasizing deviations from uniform distribution). To go around this problem, earlier one would argue epre security of the iterated hash function and directly apply the implication epre $\rightarrow \mathbf{u}$-dpre of [18]. As demonstrated, this implication does not hold, leaving this approach heuristic only.

As a fall-back option the known coll $\rightarrow \mathbf{u}$-dpre implication [18] can be used, as the generalized hash function $\mathcal{G}^{H}$ is collision secure given an injective output transformation, a suffix-free padding function pad and collision secure underlying compression function $H$. Thus we could conclude that for the hash function $\mathcal{G}^{H}$ the domain-oriented preimage advantage (based on the uniform message distribution $\mathbf{u}$ ) is at most the collision resistance of the underlying compression function (plus a vanishing error term for a sufficiently large domain). Unfortunately, this result can only ever guarantee domainoriented preimage resistance up to the birthday bound.

This is where our core result (Theorem 14 and here its cousin from Theorem 19) comes into play. We observe that since pad is injective, any distribution over $\mathcal{X}$ will lead to a distribution over $\mathcal{M}^{+}$of the same entropy. Moreover, it is guaranteed that $\ell \geq 1$ (for all messages). Thus we will define $H_{2}$ as the last compression function call in the iteration followed by $g$ and let $H_{1}$ be comprised of the remaining, earlier compression function calls (so $\ell-1$ calls for an $\ell$-block message, however we are not guaranteed all messages have equal length).

Applying Theorem 19 on a coll secure compression function $H$ and an epre secure function $H_{2}=g \circ H$, we show that $\mathcal{G}^{H}$ is $\mathbf{p}$-dpre secure for any distribution $\mathbf{p}$ of sufficient Rényi $i_{2}$-entropy. Note that the relevant epre advantage involves $g$, however if $g$ is injective it is easy to see that $H_{2}$ is at least as epre-secure as $H$.

Application to hash chains. A very similar analysis can be made for hash chains. Given a hash function $H: \mathcal{K} \times \mathcal{X} \rightarrow \mathcal{Y}$ with $\mathcal{Y} \subseteq \mathcal{X}$ the $i$ th iterate $H^{i}: \mathcal{K} \times \mathcal{X} \rightarrow \mathcal{Y}$ as $i$-fold application of $H$ (for instance, $H^{2}(K, X)=H(K, H(K, X))$ ). The properties required from hash chains are typically non-standard. For simplicity, we give informal, unkeyed description: for quasi-inversion [10] the task is, given $Y=H(X)$, to return $X^{\prime}$ and $i$ such that $H^{i}\left(X^{\prime}\right)=H^{i-1}(Y)$; for one-deeper preimage resistance the adversary should, given $Y=H^{i}(X)$, come up with $X^{\prime}$ for which $Y=H\left(X^{\prime}\right)$. The description of the latter is almost a perfect fit with ipre, all we have to do is define $H_{1}=H^{i-1}$ and $H_{2}=H$. Thus we can conclude that one-deeper preimage resistance is implied by everywhere preimage resistance and collision resistance. This suffices for the security of e.g. the Winternitz one-time signature scheme $[9,13]$. 


\section{References}

1. Andreeva, E., Neven, G., Preneel, B., Shrimpton, T.: Seven-property-preserving iterated hashing: Rox. In: Kurosawa, K. (ed.) Asiacrypt'07. LNCS, vol. 4833, pp. 130-146. Springer, Heidelberg (2007)

2. Avoine, G., Junod, P., Oechslin, P.: Characterization and improvement of time-memory tradeoff based on perfect tables. ACM Trans. Inf. Syst. Secur. 11(4) (2008)

3. Bellare, M., Kohno, T.: Hash function balance and its impact on birthday attacks. In: Cachin, C., Camenisch, J. (eds.) Eurocrypt'04. LNCS, vol. 3027, pp. 401-418. Springer, Heidelberg (2004)

4. Black, J., Rogaway, P., Shrimpton, T.: Black-box analysis of the block-cipher-based hashfunction constructions from PGV. In: Yung, M. (ed.) Crypto'02. LNCS, vol. 2442, pp. 320335. Springer, Heidelberg (2002)

5. Black, J., Rogaway, P., Shrimpton, T., Stam, M.: An analysis of the block-cipher-based hash functions from PGV. Journal of Cryptology 23(4), 519-545 (2010)

6. Brassard, G. (ed.): Crypto'89, LNCS, vol. 435. Springer, Heidelberg (1990)

7. Cachin, C.: Unconditional Security in Cryptography. Ph.D. thesis, ETH Zürich (1997)

8. Damgård, I.: A design principle for hash functions. In: Brassard [6], pp. 416-427

9. Dods, C., Smart, N., Stam, M.: Hash based digital signature schemes. In: Smart, N. (ed.) CCC'05. LNCS, vol. 3796, pp. 96-115. Springer, Heidelberg (2005)

10. Even, S., Goldreich, O., Micali, S.: On-line/off-line digital signatures. Journal of Cryptology 9(1), 35-67 (1996)

11. Hellman, M.: A cryptanalytic time-memory trade off. IEEE Transactions on Information Theory 26(4), 401-406 (1980)

12. Hevia, A., Micciancio, D.: The provable security of graph-based one-time signatures and extensions to algebraic signature schemes. In: Zheng, Y. (ed.) Asiacrypt'02. LNCS, vol. 2501, pp. 379-396. Springer, Heidelberg (2002)

13. Merkle, R.C.: A certified digital signature. In: Brassard [6], pp. 218-238

14. Merkle, R.C.: One way hash functions and DES. In: Brassard [6], pp. 428-446

15. Neven, G., Smart, N., Warinschi, B.: Hash function requirements for Schnorr signatures. Journal of Mathematical Cryptology 3(1), 69-87 (May 2009)

16. Preneel, B.: Analysis and design of cryptographic hash functions. Ph.D. thesis, Katholieke Universiteit Leuven (1993)

17. Rivest, R.L.: The md6 hash function - a proposal to nist for sha-3. Submission to NIST (2008)

18. Rogaway, P., Shrimpton, T.: Cryptographic hash-function basics: Definitions, implications and separations for preimage resistance, second-preimage resistance, and collision resistance. In: Roy, B.K., Meier, W. (eds.) FSE. LNCS, vol. 3017, pp. 371-388. Springer, Heidelberg (2004)

19. Rogaway, P.: On the role definitions in and beyond cryptography. In: Maher, M.J. (ed.) ASIAN'04. LNCS, vol. 3321, pp. 13-32. Springer, Heidelberg (2004)

20. Rogaway, P.: Formalizing human ignorance. In: Nguyen, P.Q. (ed.) VIETCRYPT'06. LNCS, vol. 4341, pp. 211-228. Springer, Heidelberg (2006)

21. Stam, M.: Blockcipher-based hashing revisited. In: Dunkelman, O. (ed.) FSE'09. LNCS, vol. 5665, pp. 67-83. Springer, Heidelberg (2009)

22. Verheul, E.R.: Selecting secure passwords. In: Abe, M. (ed.) CT-RSA'07. LNCS, vol. 4377, pp. 49-66. Springer, Heidelberg (2006) 